\title{
Resultados de la instauración de un programa de enseñanza y autocuidado en la cicatrización de úlceras venosas crónicas. Estudio de cohorte retrospectiva
}

\section{Results of the implementation of a teaching and self-care program in the healing of chronic venous ulcers. Retrospective cohort study}

\author{
Dalid R. Nieto-Zepeda, Silvia R. Flores-Aguilar, Verónica Carbajal-Robles, Amparo Quintana-Aceves, \\ Miguel Á. Sierra-Juárez y Enrique Santillán-Aguayo* \\ Servicio de Angiología y Cirugía Vascular, Hospital General de México Eduardo Liceaga, Ciudad de México, México
}

\begin{abstract}
Resumen
Antecedentes: La insuficiencia venosa crónica origina el $80 \%$ de las úlceras en las extremidades inferiores. Se ha demostrado que los programas de capacitación en la adecuada aplicación de vendajes y curación de las heridas permiten disminuir el tiempo de cicatrización, el número de recurrencias, así como las complicaciones relacionadas con el vendaje. Objetivo: Describir los efectos de un programa de entrenamiento en autocuidado y apego en la colocación correcta de vendaje compresivo. Material y métodos: Se revisaron los expedientes de los participantes del programa piloto: Entrenamiento y autocuidado en pacientes con úlceras venosas crónicas de la clínica de heridas del Servicio de Angiología y Cirugía Vascular del Hospital General de México Dr. Eduardo Liceaga. Resultados: Se analizó a 84 pacientes, de los cuales $73.8 \%$ (62) presentó cicatrización completa y el 26.2\% (22) no alcanzó cicatrización completa a las 12 semanas. El tiempo de cicatrización promedio para el total de pacientes fue de 7.6 (3.3) semanas. Conclusiones: Del total de la población estudiada se observó que el $73.8 \%$ (62) cicatrizó al final del seguimiento y que los factores de riesgo relacionados con la falta de cicatrización fueron el diámetro inicial $>5 \mathrm{~cm}$ de la úlcera y las úlceras con tiempo de evolución > 6 meses.
\end{abstract}

Palabras clave: Úlcera crónica. Insuficiencia venosa. Cicatrización. Compresión. Vendaje.

\section{Abstract}

Introduction: Chronic venous insufficiency causes $80 \%$ of ulcers in the lower extremities. It has been shown that training programs in the proper application of bandages and wound healing can reduce healing time, number of recurrences, as well as the complications associated with bandaging. Objective: to describe the impact of a training program in self-care and adherence on the correct application of a compression bandage. Materials and methods: Records of all participants of the pilot program: training and self-care in patients with chronic venous ulcers, from the wound clinic of the Angiology and Vascular Surgery Service of the Hospital General de México Dr. Eduardo Liceaga were reviewed. Results: A total of 84 patients were analyzed in the vascular wound clinic, of which $73.8 \%$ (62) showed complete healing, 26.2\% (22) did not achie-

Correspondencia:

*Enrique Santillán-Aguayo

E-mail: ensagu5@hotmail.com

0377-4740/@ 2021 Sociedad Mexicana de Angiología y Cirugía Vascular y Endovascular, A.C. Publicado por Permanyer. Este es un artículo open access bajo la licencia CC BY-NC-ND license (http://creativecommons.org/licenses/by-nc-nd/4.0/). 
ve complete healing after 12 weeks. The average healing time for all patients was 7.6 (3.3) weeks. Conclusions: Eighty-four patients were analyzed of which $73.8 \%$ (62) presented complete healing, $26.2 \%$ (22) did not reach complete healing at 12 weeks. The average healing time for all patients was 7.6 (3.3) weeks.

Key words: Chronic ulcer. Venous insufficiency. Scarring. Compression. Bandage.

\section{Introducción}

La insuficiencia venosa crónica representa el $80 \%$ de las úlceras de las extremidades inferiores y es la expresión más grave de la enfermedad venosa crónica secundaria a la hipertensión venosa constante ${ }^{1,2}$. Su naturaleza resistente la convierte en un problema de salud, ya que influye directamente en la calidad de vida y la productividad. En la actualidad se calcula que $0.06 \%$ a $2 \%$ de la población general padece úlceras crónicas en los miembros inferiores, definidas como la pérdida de continuidad de la piel por más de cuatro semanas, resistentes al tratamiento regular después de tres meses de uso continuo $0^{1,3}$.

Se han identificado diversos síntomas relacionados con la enfermedad, tanto físicos como emocionales, entre ellos dolor, piernas inquietas, alteraciones del sueño, dependencia e incluso aislamiento social; todos modifican la calidad de vida de los pacientes, algunos de los cuales representan un alto efecto económico personal y ausentismo laboral ${ }^{4-7}$.

La base fisiopatológica que predispone al desarrollo de una úlcera es la elevación de la presión venosa residual y ambulatoria, lo cual conduce a un proceso inflamatorio crónico que provoca anomalías tisulares, como depósitos de fibrina en la dermis, extravasación de líquido y separación de los espacios intercelulares, migración de leucocitos y liberación de citocinas, que perpetúan el proceso y dan lugar a la aparición de úlceras como expresión máxima de la enfermedad ${ }^{8-10}$.

Para el seguimiento es importante documentar las características clínicas de la úlcera, como localización, número, área y profundidad, características de los bordes y del lecho, propiedades del exudado e infección. Tras la institución de un tratamiento regular sin mejoría también debe considerarse la biopsia, en particular en las úlceras con más de tres meses de evolución y las dolorosas, con bordes, lecho exofítico y exudado fétido, no siempre en relación con un proceso infeccioso, para descartar degeneración maligna ${ }^{2,10,11 .}$

La base del tratamiento de las úlceras venosas crónicas es el uso de vendajes de alta compresión, que ayuda a reducir la hipertensión venosa y el edema. El promedio general de cicatrización es de $25 \%$ a $50 \%$ a las 12 semanas y de $40 \%$ a $70 \%$ si es $>12$ semanas s, $^{1,12,13}$.

En un metaanálisis en el que se incluyeron 84 estudios controlados aleatorizados, con un total de 4,321 participantes, se demostró que el tratamiento compresivo mejora la tasa de cicatrización en 12 a 16 semanas e incluso 59 días en países como Reino Unido donde se aplicaron programas de cuidado ${ }^{14-17}$.

El apego a la compresión puede variar desde 10\% hasta $80 \%$; algunos estudios demuestran que los pacientes que no tienen un adecuado apego al tratamiento compresivo muestran tiempos de cicatrización mayores y hasta 20 veces más recurrencias en comparación con los enfermos que utilizan la compresión de manera adecuada ${ }^{2,18,19}$.

En la actualidad existen datos limitados acerca de la forma en que las intervenciones educativas dirigidas a pacientes con úlceras venosas crónicas pueden mejorar el conocimiento de la enfermedad, prevenir complicaciones e incrementar el apego al tratamiento. Algunas series señalan que los folletos mejoran la observancia del tratamiento, al reducir la tasa de recurrencia hasta nueve veces, lo que representa una mejoría de la calidad de vida ${ }^{8,20}$; hay evidencia que favorece el uso de videos respecto de los folletos como método educativo y también ha registrado un incremento del cumplimiento y una disminución de las tasas de recurrencia ${ }^{8,21,22}$. En consecuencia, es importante considerar que las úlceras venosas crónicas representan enormes efectos en la calidad de vida y social, pero sobre todo en el aspecto económico, en los pacientes que la padecen y en los sistemas de salud.

\section{Material y métodos}

Se presenta una cohorte retrospectiva llevada a cabo en el Servicio de Angiología y Cirugía Vascular del Hospital General de México Dr. Eduardo Liceaga, donde se realizó una búsqueda de expedientes de los pacientes con diagnóstico de úlcera venosa crónica que participaron en el programa piloto de la clínica de heridas vasculares, para el autocuidado y entrenamiento en el uso adecuado de vendajes elastocompresivos 
para mejorar el apego al tratamiento y disminuir el tiempo de cicatrización. A todos los pacientes se les indicó de modo inicial clostridiopeptidasa/cloranfenicol, una vez que se observó tejido de granulación, y sulfadiazina de plata, con cobertura de gasas estériles. El vendaje se realizó con una venda Elastomédica de 10 $\mathrm{cm}$; se realizaron valoraciones a las semanas $0,1,4$, 8 y 12, en las que se recogió información sobre las características clínicas de las úlceras, y se introdujo un enfoque en el entrenamiento al paciente y cuidador principal acerca de la correcta colocación del vendaje compresivo, a través de un video e instrucciones en cada consulta; el video y la consulta no incluían información en relación con la enfermedad ni se consideró como parte de las variables.

La enseñanza en los pacientes y familiares inició desde la forma correcta de sostener y manipular la venda para asegurar siempre la máxima tracción, a través de movimientos simétricos que permitan ejercer una compresión uniforme sobre los tejidos. Se les indicó que controlaran con la mano dominante del colocador, con instrucciones específicas para la forma correcta de sujetar el rollo; el objetivo era mantener una tensión uniforme, algo que se consideró primordial porque, en caso contrario, si algún giro pierde tensión, también lo hace el resto de la venda; en consecuencia, junto con el video siempre se entrenó al cuidador primario de forma dirigida.

Como norma general, se liberaron dos giros sobre el dorso del pie y un tercero incluyó el talón y el tobillo, dejando el talón descubierto en mayor o menor medida (según fueran la longitud y tamaño del pie). Un cuarto giro en dirección descendente debía cubrir y fijar con firmeza el talón para suministrar mayor apoyo y presión durante la ambulación, lo cual reduce la probabilidad de que el vendaje pierda fuerza al soltarse en el tobillo.

En la pantorrilla se distribuyó en trayectos ascendentes y descendentes amplios, de tal manera que formaran un ángulo en el punto de cruce inferior menor de $60^{\circ}$, y alcanzar al final el borde inferior de la tuberosidad pretibial y distribuir el tejido faltante en el plexo sóleo y gastrocnemio con uniformidad de modo espiral, como la técnica regular (Figs. 1 y 2).

Una vez obtenidos los expedientes clínicos, se analizaron las características de los pacientes (edad, sexo, comorbilidades), así como las propiedades de las úlceras (diámetro, tiempo de evolución, granulación, área). Se creó además una fórmula para obtener el porcentaje de regeneración cutánea por semana de evaluación (Área de la úlcera en el día 1 de valoración-Área de la úlcera en el día $x$ de valoración/Área de la úlcera del día $1 \times 100$ ), se calcularon diferencias de medias de las

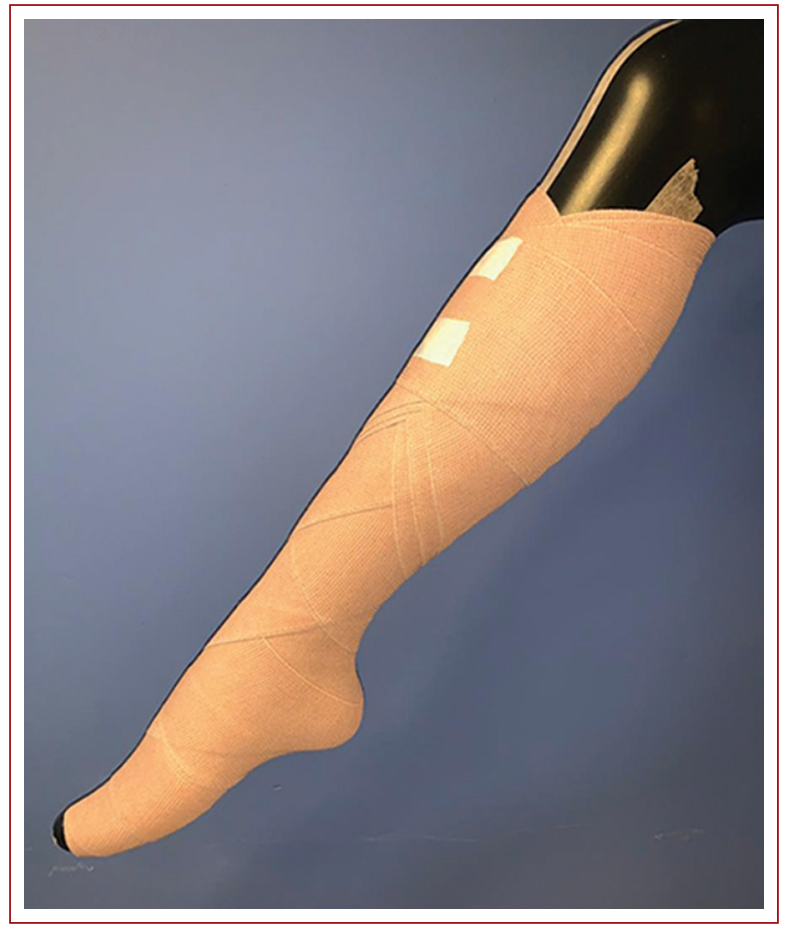

Figura 1. Resultado de la técnica de vendaje utilizada en $V$ invertida. Vista anteromedial.

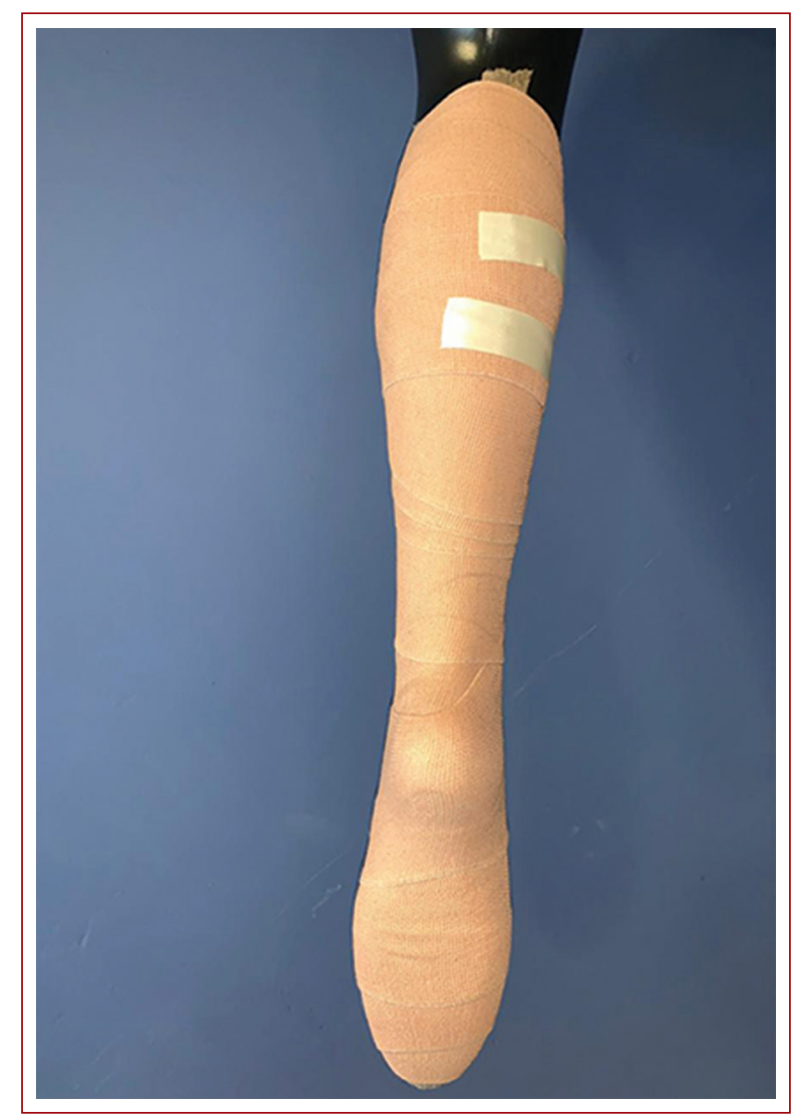

Figura 2. Resultado de la técnica de vendaje utilizada en $\mathrm{V}$ invertida. Vista posterior. 


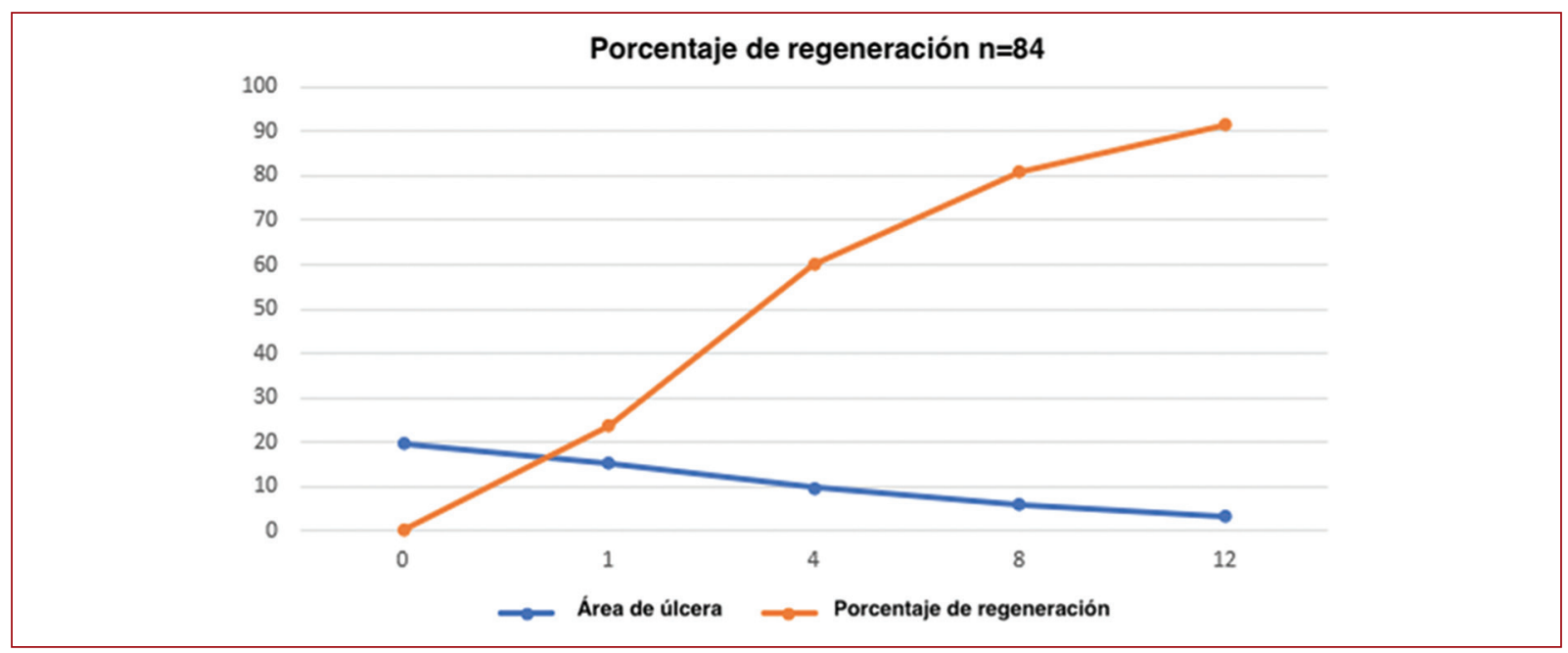

Figura 3. Cicatrización general.

características de las úlceras con prueba t de Student y para las variables cualitativas se calculó la diferencia de proporciones mediante la prueba $\chi^{2} y$ se determinaron riesgos para no cicatrizar mediante un análisis de regresión logística, tras establecer como significancia estadística un valor de $p<0.05$ y un IC95\%.

\section{Resultados}

En total se analizó a 84 pacientes en la clínica de heridas vasculares del Servicio de Angiología y Cirugía Vascular, de los cuales $73.8 \%$ (62) presentó cicatrización completa y $26.2 \%$ (22) no alcanzó cicatrización completa a las 12 semanas, pero con contracción de ésta (Fig. 3). La edad media fue de $58.6 \pm 14.3$ y de $64 \pm 15.3$ años en el grupo de cicatrización completa e incompleta, respectivamente. La comorbilidad presentada con mayor frecuencia fue la hipertensión arterial en $29 \%$ (18) y $42.9 \%$ (9), seguida de diabetes mellitus con $19.4 \%$ (12) y $19 \%$ (4) en el grupo de cicatrización completa e incompleta, respectivamente, sin diferencia significativa con una $p=0.24$ y $p=0.97$ (Tabla 1).

En el grupo de cicatrización completa se identificaron antecedentes de trombosis venosa profunda en el $14.5 \%$ (9), úlceras recidivantes en $46.8 \%$ (29), y una disminución final de $0.4 \pm 1$ en la escala visual análoga del dolor. Por su parte, en el grupo de cicatrización incompleta, la prevalencia de trombosis venosa profunda previa fue de $28.6 \%$ (6) y úlceras recidivantes de $42.9 \%$ (9), con disminución de la escala visual análoga del dolor en la medición final de $0.67 \pm 1.06$, y se hallaron diferencias significativas entre grupos con una $p=0.028$. (Tabla 1).

En cuanto a las características de las úlceras se encontró que la duración > 6 meses fue de $66.1 \%$ (41) contra $95.2 \%$ (20) en el grupo de cicatrización completa e incompleta, respectivamente, que fue mayor en el grupo que no cicatrizó, con una diferencia estadísticamente significativa de $p=0.009$. El diámetro promedio de las úlceras fue mayor en el grupo de no cicatrización con $8.21 \pm 2.6 \mathrm{~cm}$ contra $4.21 \pm 2.4 \mathrm{~cm}$ en aquellas que cicatrizaron antes de 12 semanas, con diferencia significativa de $p=0.001$. En relación con el área de la úlcera se observó una media de $12.25 \pm 11.3 \mathrm{~cm}^{3}$ en las que mostraron cicatrización completa y de $41.3 \pm 25.4 \mathrm{~cm}^{3}$ en las que no cicatrizaron con una diferencia significativa de $p=0.001$ (Tabla 2).

Respecto de las características de la piel sana, se observó una mayor prevalencia de eccema y piel seca similar en ambos grupos, sin diferencia significativa $(p=0.40)$; se notificaron complicaciones relacionadas con el vendaje en $4.8 \%$ (3) en aquellos que cicatrizaron de manera completa (Tabla 2).

El tiempo de cicatrización promedio para el total de pacientes fue de 7.6 (DE, 3.3) semanas (Tabla 3), en tanto que en el análisis del grupo de cicatrización completa fue de 7.65 (DE, 3.32) semanas; al valorar el porcentaje de regeneración en el grupo de cicatrización completa se encontró el mayor avance entre las semanas de tratamiento 1 y 4 , con $26.7 \%$ (DE, 19.7) y $68 \%$ (DE, 25.4), respectivamente, y en la semana 8 se 
Tabla 1. Características clínicas de los pacientes

\begin{tabular}{|c|c|c|c|}
\hline $\begin{array}{l}\text { Características de los pacientes } \\
\mathrm{n}=\mathbf{8 4}\end{array}$ & $\begin{array}{l}\text { Cicatrización completa } \\
n=62(73.8 \%)\end{array}$ & $\begin{array}{l}\text { Falta de cicatrización } \\
\qquad n=22(26.2 \%)\end{array}$ & $\mathbf{p}$ \\
\hline Femenino, $\mathrm{n}(\%)$ & $26(41.9)$ & $10(47.6)$ & 0.65 \\
\hline Edad media (DE) & $58.6(14.3)$ & $64(15.3)$ & 0.97 \\
\hline $\begin{array}{l}\text { Comorbilidades, } n(\%) \\
\text { HTA } \\
\text { DM } \\
\text { TVP } \\
\text { Fracturas MP } \\
\text { Enfermedades autoinmunitarias }\end{array}$ & $\begin{array}{c}18(29) \\
12(19.4) \\
9(14.5) \\
7(11.3) \\
2(3.2)\end{array}$ & $\begin{array}{c}9(42.9) \\
4(19) \\
6(28.6) \\
0 \\
3(14.3)\end{array}$ & $\begin{array}{l}0.24 \\
0.97 \\
0.14 \\
0.11 \\
0.06\end{array}$ \\
\hline Operación venosa previa, n (\%) & $7(11.3)$ & $3(14.3)$ & 0.71 \\
\hline Úlcera recidivante, n (\%) & $29(46.8)$ & $9(42.9)$ & 0.75 \\
\hline Úlcera bilateral, n (\%) & $6(9.7)$ & $2(9.5)$ & 0.98 \\
\hline EVA inicial media (DE) & $3.6(2.3)$ & $2.8(2.06)$ & 0.019 \\
\hline EVA final media (DE) & $0.4(1)$ & $0.67(1.06)$ & 0.028 \\
\hline $\begin{array}{l}\text { Arco de movilidad, } \mathrm{n}(\%) \\
0 \\
1 \\
2 \\
3 \\
4\end{array}$ & $\begin{array}{c}2(3.2) \\
1(1.6) \\
15(24.59) \\
19(31.14) \\
25(40.23)\end{array}$ & $\begin{array}{c}0 \\
0 \\
0 \\
8(38.1) \\
13(61.9)\end{array}$ & 0.48 \\
\hline
\end{tabular}

Diferencia de medias con $t$ de Student, diferencia de proporciones con $\chi^{2}, \mathrm{P}<0.05$, IC95\%

HTA: hipertensión arterial; DM: diabetes mellitus; TVP: trombosis venosa profunda; MP: miembros pélvicos; EVA: escala visual análoga del dolor.

Tabla 2. Características de las úlceras

\begin{tabular}{|c|c|c|c|}
\hline $\begin{array}{l}\text { Características de las úlceras } \\
\mathrm{n}=84\end{array}$ & $\begin{array}{c}\text { Cicatrización completa } \\
n=62(73.8 \%)\end{array}$ & $\begin{array}{l}\text { Falta de cicatrización } \\
\qquad n=22(26.2 \%)\end{array}$ & $\mathbf{p}$ \\
\hline Úlceras > 6 meses, n (\%) & $41(66.1)$ & $20(95.2)$ & 0.009 \\
\hline Diámetro de úlcera, cm media (DE) & $4.21(2.4)$ & $8.21(2.6)$ & 0.001 \\
\hline Área de la úlcera, cm2 media (DE) & $12.25(11.3)$ & $41.3(25.4)$ & 0.001 \\
\hline $\begin{array}{l}\text { Piel circunferencial, n (\%) } \\
\text { Sana } \\
\text { Seca } \\
\text { Eccema } \\
\text { Eritema }\end{array}$ & $\begin{array}{c}13(21) \\
26(41.9) \\
20(32.3) \\
3(4.8)\end{array}$ & $\begin{array}{c}2(9.5) \\
7(33.3) \\
10(47.6) \\
2(9.5)\end{array}$ & 0.40 \\
\hline $\begin{array}{l}\text { Granulación, n (\%) } \\
\quad<25 \% \\
26-50 \% \\
51-75 \% \\
>76 \%\end{array}$ & $\begin{array}{c}7(11.3) \\
33(53.2) \\
17(27.4) \\
5(8.1)\end{array}$ & $\begin{array}{c}10(47.6) \\
8(38.1) \\
2(9.5) \\
1(4.8)\end{array}$ & 0.01 \\
\hline Complicaciones del vendaje $\mathrm{n}(\%)$ & $3(4.8)$ & 0 & 0.99 \\
\hline
\end{tabular}

Diferencia de medias con t de Student, diferencia de proporciones con $\chi^{2}, \mathrm{P}<0.05, \mathrm{IC} 95 \%$.

observó un $90.2 \%$ (DE, 16.3) de regeneración y $100 \%$ a las 12 semanas.

Se efectuó un análisis de riesgo para identificar factores de riesgo para no lograr cicatrización a las 12 semanas al final del seguimiento y se identificó como factor protector el tiempo de evolución de las úlceras $<6$ meses con un $\mathrm{HR}=0.4(\mathrm{IC}, 0.2-0.7 ; \mathrm{p}=0.01)$, y la presencia de un diámetro de las úlceras $<5 \mathrm{~cm}$ con 
Tabla 3. Cicatrización general

\begin{tabular}{|l|c|c|c|c|c|}
\hline \multicolumn{7}{|c|}{ Cicatrización general, $\mathbf{n = 8 4}(\mathbf{1 0 0} \%)$} \\
\hline Tiempo promedio de cicatrización media (DE) \\
\hline Semanas & 0 & 1 & $7.6(3.3)$ & 12 \\
\hline Área de úlcera media (DE) & $19.6(21.3)$ & $15.3(16.2)$ & $9.6(12.4)$ & $5.6(9.2)$ & $3.1(7)$ \\
\hline Porcentaje de regeneración media (DE) & 0 & $23.6(19.3)$ & $60.1(27.6)$ & $80.8(24.02)$ & $91.6(17.4)$ \\
\hline
\end{tabular}

Tabla 4. Riesgo de falta de cicatrización a las 12 semanas

\begin{tabular}{|l|c|c|c|}
\hline Factor & HR & IC (95\%) & $p$ \\
\hline Edad & 1.01 & $0.98-1.0$ & 0.25 \\
\hline Género & 0.9 & $0.3-2.2$ & 0.8 \\
\hline DM & 0.6 & $0.1-2.3$ & 0.4 \\
\hline HTA & 1.6 & $0.5-4.5$ & 0.3 \\
\hline TVP & 1.4 & $0.5-4.2$ & 0.4 \\
\hline Fractura MP & 0.4 & $0-5.2$ & 0.3 \\
\hline Úlcera recidivante & 1.2 & $0.4-2.9$ & 0.6 \\
\hline Úlcera bilateral & 1.04 & $0.2-5.3$ & 0.96 \\
\hline Arco de movilidad & 1.0 & $0.5-1.7$ & 0.9 \\
\hline Tiempo de úlcera $<6 \mathrm{~m}$ & 0.4 & $0.2-0.7$ & 0.01 \\
\hline Área inicial & 0.98 & $0.9-1.01$ & 0.26 \\
\hline Diámetro < $5 \mathrm{~cm}$ & 0.7 & $0.6-0.9$ & 0.03 \\
\hline
\end{tabular}

DM: diabetes mellitus; HTA: hipertensión arterial; TVP: trombosis venosa profunda; MP miembros pélvicos.

un $\mathrm{HR}=0.7$ (IC, 0.6-0.9; $\mathrm{p}=0.03)$. Las demás variables analizadas como antecedentes patológicos no mostraron estadísticas significativas (Tabla 4).

\section{Discusión}

Las úlceras secundarias a insuficiencia venosa crónica ocasionan un gran efecto económico en los sistemas de salud, con tasas elevadas de incapacidad, y disminución de la calidad de vida ${ }^{4,5}$; en consecuencia, se creó un programa piloto en la clínica de heridas vasculares del Servicio de Angiología y Cirugía Vascular, con la finalidad de entrenar a los pacientes y familiares en la correcta colocación y adecuado uso del vendaje compresivo, con una técnica específica y estandarizada diseñada por el servicio denominada en $\mathrm{V}$ invertida. Se incluyó a un total de 84 pacientes con un seguimiento de 12 semanas, de los cuales 62 (73.8\%) experimentaron cicatrización completa y $22(26.2 \%)$ no cicatrizaron al final del seguimiento, lo que contrasta con los porcentajes informados en diversos estudios con un promedio general de cicatrización de $25 \%$ a $50 \%$ antes de 12 semanas y de $40 \%$ a $70 \%$ después de 12 semanas ${ }^{1,5,12,13}$.

Se encontró mayor prevalencia del sexo masculino en ambos grupos, a diferencia de lo informado en las publicaciones médicas en las que se menciona una relación que varía de 1.5:1 a 10:1 para el sexo femenino ${ }^{1,2}$; en cuanto a la edad, diversos estudios indican que la mayoría de los pacientes presentaría una primera úlcera entre los 40 y 60 años, lo cual concuerda con los pacientes analizados, con una edad media de 61.3 años (DE, 14.5 años).

En cuanto a las comorbilidades notificadas en las publicaciones médicas se ha observado la elevada prevalencia de diabetes mellitus e hipertensión arterial como factores de riesgo para la falta de cicatrización; sin embargo, en este estudio no se identificaron diferencias significativas entre los grupos de acuerdo con las comorbilidades. Los factores relacionados e informados como deletéreos en diferentes estudios y con los que se ha observado mayor riesgo de falta de cicatrización incluyen úlcera recidivante y trombosis venosa previa, los cuales resultaron no significativos en este estudio, junto con un tiempo de evolución de la úlcera $>6$ meses y un diámetro $>5 \mathrm{~cm}$, que en este grupo de falta de cicatrización fueron los motivos más frecuentes, con una diferencia estadísticamente significativa $(p<0.05)$.

Sánchez-Nicolat, et al. en un estudio de revisión encontraron que los factores más vinculados con el retraso de la cicatrización son el tiempo de evolución de las úlceras, el antecedente de operación venosa, operación para reemplazo de cadera, úlceras con más de $50 \%$ de cobertura de fibrina, tiempo de evolución y tamaño de la úlcera. En este estudio se llevó a cabo un análisis de riesgo multivariado para identificar estos factores de 
riesgo y se encontraron como únicos factores significativos el tiempo de evolución de la úlcera $>6$ meses y el diámetro $>5 \mathrm{~cm}$, lo cual podría explicarse por alteraciones en la angiogénesis y migración celular detenida, que junto al edema por hipertensión venosa ocasiona disminución de la actividad de los miofibroblastos y la producción de colágeno. La ausencia de significancia estadística del resto de los factores de riesgo se explica probablemente por el número de la población, dado que se trata de un estudio piloto en el que se realizó la intervención en todos los participantes, y la posible exclusión de otras variables, además de que algunos pacientes se perdieron en el seguimiento (92 totales, eliminados $8, n=84$ ); por lo tanto, la comparación es limitada.

Es importante mencionar el potencial papel de la limitación de la movilidad de la articulación del tobillo, ya que el $61.9 \%$ de los pacientes que no lograron cicatrizar antes de 12 semanas presentaba una reducción de los arcos de movilidad $>10^{\circ}$, en comparación con un $40.23 \%$ en el grupo que logró cicatrizar; no obstante, al efectuar el análisis de riesgo no se encontró un valor significativo $(p=0.9)$ (Tabla 4).

Se evaluó el dolor de acuerdo con la escala visual análoga al inicio y al final del seguimiento y se identificó una mayor reducción del dolor con una diferencia significativa en todos los pacientes $(p=0.019$ inicial $y$ $p=0.028$ final).

En todos los estudios se ha demostrado que la base del tratamiento de las úlceras venosas debe enfocarse en la terapia compresiva, además de su colocación por personal entrenado o capacitación de manera adecuada al paciente para garantizar un buen apego, pero la mayor parte de los estudios se inclina en favor de los vendajes multicapa. A diferencia de este estudio, en el que no se incluyeron múltiples capas, se logró un apego del $100 \%$ a la terapia compresiva; esto se debió a que se le dio un seguimiento periódico semanal en el que se insistía en la técnica de colocación. Las complicaciones relacionadas con el vendaje fueron sólo del $4.8 \%$, similar a lo notificado en las publicaciones médicas y el porcentaje de cierre de las heridas a las 12 semanas fue del $73.8 \%$, similar a lo informado en los diferentes estudios con sistemas multicapa, a través de una técnica de colocación que, en la experiencia de los autores, podría considerarse multicapa debido a la forma de la distribución.

El objetivo principal de este estudio piloto fue evaluar los resultados de una intervención educativa, a través de una técnica de colocación de vendaje estandarizada, y se observó que en las publicaciones médicas los datos son limitados acerca de la forma en que estas intervenciones pueden mejorar el conocimiento sobre la enfermedad y el apego al tratamiento.

\section{Conclusiones}

Las limitaciones halladas en este estudio fueron el tamaño de la población, la cual debe ampliarse; además, hay que aumentar el tiempo de seguimiento y presentar la estandarización de la técnica utilizada con medidas objetivas, como escalas de calidad de vida, medición de la presión de subvendaje y la pletismografía para el cálculo de la fracción de expulsión de la pantorrilla, para compararla con las técnicas de vendaje ya descritas actualmente, y con las prendas compresivas. Los autores se enfocaron en un programa con estrecho seguimiento en el que se le enseñó al paciente y a sus cuidadores la correcta aplicación del vendaje, para demostrar al final la obtención de un adecuado porcentaje de cicatrización al término del seguimiento (73.8\%), con beneficios comparables a los resultados en sistemas multicapa.

Del total de la población estudiada en el programa piloto de entrenamiento en autocuidado y apego en la colocación correcta del vendaje compresivo en pacientes con úlceras de origen venoso en miembros pélvicos, se encontró que el $78.3 \%$ (62) alcanzó una cicatrización completa al final del seguimiento; los factores de riesgo relacionados para la falta de cicatrización al final del seguimiento fueron el diámetro inicial $>5 \mathrm{~cm}$ de la úlcera y las úlceras con tiempo de evolución $>6$ meses, ambas estadísticamente significativas. En consecuencia, se propone la creación de clínicas de compresión orientadas al cuidado y seguimiento en la atención avanzada de heridas.

\section{Financiamiento}

Esta investigación no ha recibido ninguna beca específica de agencias de los sectores públicos, comercial, o sin ánimo de lucro.

\section{Conflicto de intereses}

Los autores declaran no tener conflicto de intereses.

\section{Responsabilidades éticas}

Protección de personas y animales. Los autores declaran que para esta investigación no se han realizado experimentos en seres humanos ni en animales. 
Confidencialidad de los datos. Los autores declaran que han seguido los protocolos de su centro de trabajo sobre la publicación de datos de pacientes.

Derecho a la privacidad y consentimiento informado. Los autores han obtenido el consentimiento informado de los pacientes o sujetos referidos en el artículo. Este documento obra en poder del autor de correspondencia.

\section{Bibliografía}

1. Sánchez-Nicolat NE, Guardado-Bermúdez F, Arriaga-Caballero JE, Torres-Martínez JA, Flores-Escartín M, Serrano-Lozano JA, et al. Revisión en úlceras venosas: epidemiología, fisiopatología, diagnóstico y tratamiento actual. Rev Mex Angiol. 2019;47(1):26-38.

2. O'Donnell TF, Passman MA, Marston WA, Ennis WJ, Dalsing M, Kistner $R L$, et al. Management of venous leg ulcers: clinical practice guidelines of the Society for Vascular Surgery ${ }^{\circledR}$ and the American Venous Forum. J Vasc Surg. 2014;60(2):3S-59S.

3. Fernandes Abbade LP, Lastória S. Venous ulcer: epidemiology, physiopathology, diagnosis and treatment. Int J Dermatol. 2005;44(6):449-56.

4. Guest JF, Fuller GW, Vowden P. Venous leg ulcer management in clinica practice in the UK: costs and outcomes. Int Wound J. 2018;15(1):29-37.

5. Cheng Q, Gibb M, Graves N, Finlayson K, Pacella RE. Cost-effectiveness analysis of guideline-based optimal care for venous leg ulcers in Australia. BMC Health Serv Res. 2018;18(1):1-13.

6. Guest JF, Gerrish A, Ayoub N, Vowden K, Vowden P. Clinicaoutcomes and costeffectiveness of three alternative compression systems used in the management of venous leg ulcers. J Wound Care. 2015;24(7):300-10

7. Heinen M, Borm G, Van der Vleuten C, Evers A, Oostendorp R Van Achterberg T. The Lively Legs self-management programme increased physical activity and reduced wound days in leg ulcer patients: results from a randomized controlled trial. Int J Nurs Stud. 2012;49(2):151-61.

8. Santillán-Aguayo E, Carbajal-Robles V, Córdova-Quintal P, Lecuona-Huet N, De La Rosa-Bandini A, Fabián-Mijangos W, et al. Incidencia de degeneración maligna en úlceras venosas crónicas en el Servicio de Angiología y Cirugía Vascular del Hospital General de México "Dr. Eduardo Liceaga". Rev Mex Angiol. 2016;44(1):14-20.
9. Chamanga ET. Understanding venous leg ulcers. Br J Community Nurs. 2018;23(September):S6-15.

10. Raffetto JD. Pathophysiology of chronic venous disease and venous ulcers. Surg Clin North Am. 2018;98(2):337-47.

11. Lim CS, Baruah M, Bahia SS. Diagnosis and management of venous leg ulcers. BMJ. 2018;362(August):1-9.

12. Andriessen A, Apelqvist J, Mosti G, Partsch H, Gonska C, Abel M. Compression therapy for venous leg ulcers: risk factors for adverse events and complications, contraindications - a review of present guidelines. J Eur Acad Dermatology Venereol. 2017;31(9):1562-8.

13. Guest JF, Charles H, Cutting KF. Is it time to re-appraise the role of compression in non-healing venous leg ulcers? J Wound Care. 2013; 22(9):453-60.

14. O'Meara S, Cullum N, Nelson EA, Dumville JC. Compression for venous leg ulcers (Review). Cochrane Libr. 2012;(11).

15. Atkin L, Schofield A, Kilroy-Findley A. Updated leg ulcer pathway: improving healing times and reducing costs. Br J Nurs. 2019; 28(20):21-6.

16. Martinengo L, Yeo NJY, Markandran KD, Olsson M, Kyaw BM, Car LT. Digital health professions education on chronic wound management: a systematic review. Int J Nurs Stud. 2020;104:103512.

17. Alavi A, Sibbald RG, Phillips TJ, Miller OF, Margolis DJ, Marston W, et al. What's new: management of venous leg ulcers Approach to venous leg ulcers. J Am Acad Dermatol. 2016;74(4):627-40.

18. O'meara S, Cullum N, Nelson EA, Dumville JC. Compression for venous leg ulcers. Cochrane Database Syst Rev. 2012;2012(11).

19. Carvalho M, Olivera BB. Terapia compresiva para el tratamiento de úlceras venosas: una revisión sistemática de la literatura. Terapia compressiva para o tratamento de úlceras venosas : uma revisão sistemática da literatura Compression therapy for venous leg ulcers: a systematic. Enfermería Glob. 2015;45(0):574-93.

20. Protz K, Dissemond J, Seifert M, Hintner M, Temme B, Verheyen-Cronau $\mathrm{I}$, et al. Education in people with venous leg ulcers based on a brochure about compression therapy: a quasi-randomised controlled trial. Int Wound J. 2019;16(6):1252-62.

21. Weller C, Buchbinder R, Johnston R. Interventions for helping people adhere to compression treatments for venous leg ulceration (Review). Cochrane Database Syst Rev. 2016;(3):1-47.

22. Baquerizo Nole KL, Yim E, Van Driessche F, Lamel SA, Richmond NA, Braun LR, et al. Educational interventions in venous leg ulcer patients. Wound Repair Regen. 2015;23(1):137-40. 